\title{
Performance of Coded Multicarrier Multiple Transmit Antenna DS-CDMA Systems in the Presence of Power Amplifier Nonlinearity
}

\author{
K. R. Shankar kumar and A. Chockalingam \\ Department of Electrical Communication Engineering \\ Indian Institute of Science, Bangalore 560012
}

\begin{abstract}
While a multicarrier approach of achieving frequency diversity performs well in the presence of jamming, it suffers from the effects of intermodulation distortion (IMD) due to power amplifier (PA) nonlinearity. On the other hand, transmit diversity using multiple transmit antennas has the benefit of no IMD effects, but suffers from a larger performance degradation due to jamming compared to the multicarrier approach. Hence, hybrid diversity schemes which use both multicarrier as well as multiple transmit antennas are of interest. Techniques to suppress IMD effects in such hybrid diversity schemes are important. In this paper, we propose a minimum mean square error (MMSE) receiver to suppress the intermodulation distortion in a coded multicarrier multiple transmit antenna ( $P$ transmit antennas) DS-CDMA system with $M$ subcarriers on each transmit antenna. The system uses rate- $1 / M$ convolutional coding, interleaving and space-time coding. We compare the performance of a $(M=4, P=2)$ scheme and a $(M=2, P=4)$ scheme, both having the same diversity order. We show that the proposed MMSE receiver effectively suppresses the IMD effects, thus enabling to retain better antijamming capability without much loss in performance due to IMD effects.
\end{abstract}

Keywords - Multicarrier DS-CDMA, multiple transmit antennas, interference suppression, MMSE receiver, intermodulation distortion.

\section{INTRODUCTION}

Broadband communication using code division multiple access (CDMA) can be achieved either by using a single carrier wideband direct-sequence (DS) waveform or by using several disjoint, narrowband DS waveforms on multiple subcarriers. Multicarrier (MC) approach offers several advantages including robustness in fading and interference, operation at lower chip rates (and hence lower device clock speeds and device power consumption), and non-contiguous bandwidth operation. Several studies have analyzed the performance of multicarrier DS-CDMA systems under various scenarios [1][4]. While the multicarrier approach offers frequency diversity benefits and performs well under jamming conditions, a main concern with it is the of intermodulation distortion (IMD) and harmonic distortion due to nonlinearity of the power amplifier (PA) [5]. More recently, the use of multiple transmit antennas as a means to achieve (transmit) diversity has gained increased importance owing to its ability to considerably increase the data rates on wireless fading channels [6]-[8]. Accordingly, multicarrier schemes with multiple transmit antennas have been investigated in recent studies [9],[10]. In [9], multiple transmit antennas have been used with a motivation

This work was supported in part by the Swamajayanti Fellowship from the Department of Science and Technology, Government of India, New Delhi, under scheme Ref: No, 6/3/2002-S.F to remove the correlation between the different subcarriers in a multicarrier system. In [10], we have shown that, in order to achieve a given system diversity order, a hybrid diversity approach which uses both multicarrier as well as multiple transmit antennas is superior compared to a pure multicarrier approach (frequency diversity alone) or a pure multiple transmit antennas approach (transmit diversity alone), particularly in the presence of both PA nonlinearity as well as jamming. Techniques to suppress intermodulation distortion in such hybrid diversity schemes can further improve performance. In this paper, we propose a minimum mean square error (MMSE) receiver to suppress the intermodulation distortion effects in a coded multicarrier multiple transmit antenna DS-CDMA system with $M$ subcarriers on each transmit antenna. The system uses rate- $1 / M$ convolutional coding, interleaving and spacetime block coding. We show that the proposed MMSE receiver effectively suppresses the IMD while retaining better antijamming performance.

The rest of the paper is organized as follows. In Sec. II, the system model including the multicarrier, multiple transmit antenna DS-CDMA transceiver and the nonlinear PA model are. presented. Sec. III presents the proposed MMSE receiver for the system considered. Sec. IV presents the coded bit error rate (BER) performance results. Conclusions are presented in Sec. V.

\section{SYSTEM MODEL}

We consider a coded multicarrier DS-CDMA system with $K$ users, each having $P$ transmit antennas with $M$ subcarriers on each transmit antenna. Here, we describe the system model for the case of two transmit antennas (i.e., $P=2$ ) for simplicity ${ }^{1}$. Fig. 1 shows the transmitter with two transmit antennas. The information bits are first encoded by a rate- $1 / M$ convolutional coder, the output of which is interleaved, space-time coded and mapped on to the two transmit antennas. On each transmit antenna path, a multicarrier modulator (with $M$ subcarriers) followed by a nonlinear power amplifier is employed.

The space-time coding used [7] is explained as follows. Let $b_{1}^{(k)}$ and $b_{2}^{(k)}$ represent two consecutive bits of the $k$ th user. Let $T$ represent one bit interval. Let $\mathbf{u}^{(k)}=\left\{u_{1}^{(k)}, u_{2}^{(k)}, \ldots, u_{M}^{(k)}\right\}$ and $\mathbf{v}^{(k)}=\left\{v_{1}^{(k)}, v_{2}^{(k)}, \ldots, v_{M}^{(k)}\right\}$ denote the coded and interleaved symbol vectors corresponding to the bit intervals of $b_{1}^{(k)}$ and $b_{2}^{(k)}$, respectively. During time interval 0 to $T$, the

${ }^{1}$ Extension of the model for $P>2$ (up to $P=8$ ) using orthogonal spacetime codes [8] is straightforward. 


\section{Communications - // /811}

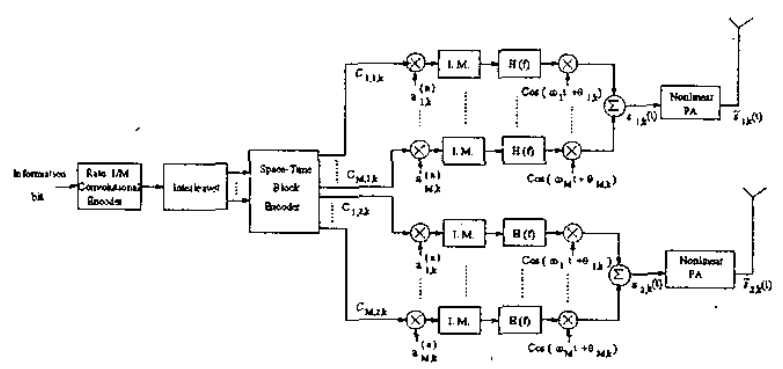

Fig. 1. Multicarrier DS-CDMA transmitter with two transmit antennas.

\begin{tabular}{|c|c|c|}
\hline & Tx Antenna 1 & Tx Antenna 2 \\
\hline time $t$ & $\mathbf{u}^{(k)}$ & $\mathbf{v}^{(k)}$ \\
\hline time $t+T$ & $\mathbf{v}^{(k)}$ & $-\mathbf{u}^{(k)}$ \\
\hline
\end{tabular}

TABLE I

SPACE-TIME TRANSMISSION SEQUENCE ON 2 TX ANTENNAS OF USER $\boldsymbol{k}$.

space-time encoder transmits $\mathbf{u}^{(k)}$ on transmit antenna 1 and $\mathbf{v}^{(k)}$ on transmit antenna 2. During time interval $T$ to $2 T$, the space-time encoder transmits $\mathbf{v}^{(k)}$ on antenna 1 and $-\mathbf{u}^{(k)}$ on antenna 2 (see Table I). On each transmit antenna path, a multicarrier modulator with $M$ disjoint subcarriers is used. The bandwidth on each of these subcarriers is $W / M$, where $W$ is the system bandwidth. The $m$ th element in a spacetime coded symbol vector modulates the $m$ th subcarrier, after being multiplied by $m$ th spreading sequence of the $k$ th user $a_{m, k}, m=1,2, \ldots, M, k=1,2, \ldots, K$.

The signal at the output of the multicarrier modulator on the $p$ th-transmit antenna path of the $k$ th user, $s_{p, k}(t), p=1,2$, $k=1,2, \ldots, K$, is given by

$$
s_{p, k}(t)=A \sum_{i=-\infty}^{\infty} \sum_{m=1}^{M} c_{m, p, k}^{(i)} f_{m, k}\left(t-i T-\tau_{k}\right) \cos \left(\omega_{m} t+\theta_{m, k}\right),(1)
$$

where $A$ is the signal amplitude, $\dot{c}_{m, p, k}^{(i)} \in\{ \pm 1\}$ represents the data stream that modulates the $m$ th subcarrier of the $p$ th transmit antenna of the $k$ th user, $\omega_{m}$ is the $m$ th subcarrier frequency, $\tau_{k}$ is an arbitrary time delay of the $k$ th user, uniformly distributed in $[0, T], \theta_{m}, k$ represents the random phase of the $m$ th subcarrier of the $k$ th user, uniformly distributed in $[0,2 \pi]$, and $f_{m, k}(t)$ is a spreading waveform given by

$$
f_{m, k}(t)=\sum_{n=0}^{N-1} a_{m, k}^{(n)} h\left(t-n M T_{c}\right)
$$

where $a_{m, k}^{(n)} \in\{ \pm 1\}$ is the $n$th chip of the spreading sequence on the $m$ th subcarrier of the $k$ th user, $N$ is the processing gain (which is taken to be equal to the period of the spreading sequence), $h(t)$ is the impulse response of the chip shaping filter, and $1 / T_{c}$ is the chip rate of a single-carrier (SC) DS-CDMA system that occupies the same spread bandwidth $W$ as does the multicarrier system, i.e., $T=N M T_{c}$. The output of the multicarrier modulator, $s_{p, k}(t)$, drives the power amplifier. Since the PA is nonlinear and at its input is the sum of the modulated signals on the $M$ subcarriers, the PA output will contain intermodulation and harmonic terms.

\section{A. Nonlinear PA output}

If the input signal to the PA is the sum of $M$ subcarriers with equally spaced frequencies (i.e., the $m$ th subcarrier frequency $\omega_{m}=\omega_{o}+m \Delta \omega, m=1,2, . ., M$, where $\Delta \omega$ is the frequency separation between two consecutive subcarriers), then the signal at the output of the PA, $g(t)$, can be written as [5]

$$
\begin{gathered}
g(t)=\operatorname{Re}\left\{\exp \left(j \omega_{0} t\right) \cdot \sum_{\substack{I_{1}, I_{2}, \ldots, I_{M}=-\infty \\
\left(I_{1}+I_{2}+\ldots+I_{M}=1\right)}}^{\infty} \operatorname{ex}\left(j \sum_{m=1}^{M}\left(m \Delta \omega t+\theta_{m}\right) I_{m}\right)\right. \\
\left.\cdot G\left(t_{i} I_{1}, I_{2}, \ldots, I_{M}\right)\right\} .
\end{gathered}
$$

where $I_{1}, I_{2}, \ldots, I_{M}$ can take integer values such that the pattern $\left\{I_{1}, I_{2}, \ldots, I_{M}\right\}$ indicates a specific tone with frequency $\left(\omega_{o}+I_{1} \Delta \omega+2 I_{2} \Delta \omega+3 I_{3} \Delta \omega+\ldots+I_{M} M \Delta \omega\right)$ at the output. These output tones (corresponding to different tone patterns) whose frequencies fall outside the system bandwidth, $W$, can be ignored, and only those tones whose frequencies fall within $W$ are of interest. Also, the condition $I_{1}+I_{2}+\ldots+I_{M}=1$ in (3) implies that only the odd-order IMD terms are considered. Further, only the 3rd- and 5th-order IMD terms are normally considered as higher odd-order IMD term amplitudes are very small. The term $G\left(t ; I_{1}, I_{2}, \ldots, I_{M}\right)$ in (3) represents the amplitude of the output tone due to the tone pattern $\left\{I_{1}, I_{2}, \ldots, I_{M}\right\}$, which can be approximated as [5]

$$
G\left(t_{i} I_{1}, I_{2}, \ldots, I_{M}\right)=\sum_{s=1}^{S} \nu_{s} \prod_{m=1}^{M} J_{I_{m}}\left(s \epsilon \bar{A}_{m}(t)\right)
$$

where $A_{m}(t)$ is the signal amplitude of the $m$ th subcarrier, $J_{n}()$ is the Bessel function of the first kind and $n^{t h}$ order, $\nu_{s}, s=1,2, . S$ represent the complex coefficients in the expansion having $S$ terms (taken to be 10 ), and $0<\epsilon<1$ [5]. From (1), the instantaneous amplitude of the $m$ th subcarrier signal at the $n$th chip in the $i$ th symbol on the $p$ th transmitting antenna of the $k$ th user is given

$$
\tilde{A}_{m, p, k}\left(i T+n \dot{M} T_{c}\right)=A h^{(n, i)} c_{m, p, k}^{(i)} a_{m, k}^{(n)}
$$

where $h^{(i, n)}$ is the impulse response of the chip shaping filter sampled at the chip interval. By substituting the amplitude in (5) and the corresponding phase of the input signal into (3), and ignoring the higher order IMD terms other than $3 \mathrm{rd}$ - and 5th-order terms, the signal at the output of the PA can be obtained as

$$
\begin{aligned}
& \tilde{s}_{p, k}(t)=\sum_{i=-\infty}^{\infty} \sum_{m=1}^{M} X_{I(m), p, k}^{(i)}(t)+X_{3(m), p, k}^{(i)}(t)+X_{5(m), p, k}^{(i)}(t),(6) \\
& x_{I(m), p, k}^{(i)}(t)=c_{I(m), p, k}^{(i)} \sum_{\{I(m)\}} \tilde{p}_{I(m), k}^{\left(t-i T-\tau_{k}\right) \cos \left(\omega_{m} t+\theta_{I(m), k}\right),(7)}
\end{aligned}
$$

where $\{I(m)\}, I=1,3,5$, represents the sets which form all patterns of $I$-th order IMD on the $m$ th subcarrier, and

$$
\tilde{p}_{I(m), k}(t)=\sum_{n=0}^{N-1} a_{I(m), k}^{(n)} h_{I(m)}\left(t-n M T_{c}\right),
$$

where $a_{I(m), k}$ is the equivalent intermodulation chip sequence corresponding to the tone pattern $I(m)$. For example, if the 


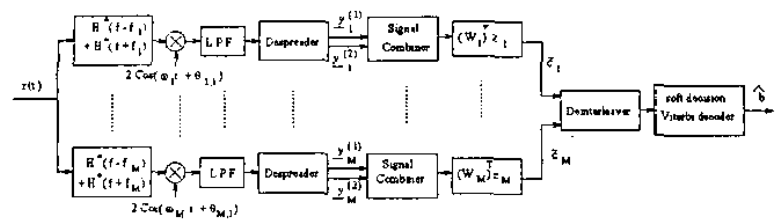

Fig. 2. Receiver for multicarrier DS-CDMA with two transmit antennas

tone pattern is $\{0,2,-1,0\}$, then the corresponding $a_{I(m), k}$ is given by $a_{2, k} a_{2, k} a_{3, k}$. For a rectangular chip shaping filter, $h_{I(m)}(t)=G_{I(m)}(t)$ computed from (4).

\section{B. Signal Combining at the Receiver}

We assume that the channel fading is independent from one subband to the other and is frequency non-selective within each subband. We further assume that the fade remains constant over the quasi-static interval of $2 T$ time units. The channel transfer function for the $m$ th subband on the $p$ th transmit antenna of the $k$ th user is given by $A_{m, p, k}=$ $\alpha_{m, p, k} \exp \left(j \beta_{m, p, k}\right)$, where the $\left\{\alpha_{m, p, k}\right\}$ are assumed to be i.i.d Rayleigh r.v's with a unit second moment, and the $\left\{\beta_{m, p, k}\right\}$ are i.i.d uniform r.v's in $[0,2 \pi]$. We also assume that the signal gets jammed by a narrowband jamming signal. We consider one receive antenna at the receiver (although the model is easily extended to any number of receive antennas). The received signal can be written as

$$
\begin{array}{r}
r(t)=\sum_{k=1}^{K} \sum_{p=1,2} \sum_{i=-\infty}^{\infty} \sum_{m=1}^{M}\left(\tilde{X}_{1(m), p, k}^{(i)}(t)+\tilde{X}_{3(m), p, k}^{(i)}(t)\right. \\
\left.+\tilde{X}_{5(m), p, k}^{(i)}(t)\right)+(t)+(\phi),
\end{array}
$$

where

$$
\begin{gathered}
\tilde{X}_{I(m), p, k}^{(i)}(t)=A_{m, p, k} c_{I(m), p, k}^{(i)} \sum_{i I(m)\}} \tilde{p}_{I(m), k}\left(t-i T-\tau_{k}\right) \\
\cdot \cos \left(\omega_{m} t+\theta_{I(m), k}\right)
\end{gathered}
$$

\section{$n_{w}(t)$ is AWGN with two-sided psd of $\eta_{6} 2$.}

We assume that user 1 is the desired user whose timing and channel coefficients are perfectly known at the receiver. The receiver for the desired user is shown in Fig. 2. The signal combining at the receiver for the space-time coded transmission. with two transmit antennas is done as follows. Consider the chip sampled output at the output of the despreader for the $m$ th subcarrier during $i$ th symbol. Let $\mathbf{y}_{m}^{(1)}, \mathbf{y}_{m}^{(2)}$ denote the $N$-chip vector output of the despreader of the $m$ th subcarrier during the first and second time slots (i.e., 0 to $T$ and $T$ to $2 T$ ). These two slot outputs are linearly combined (space-time decoding) to generate $N$-chip vector outputs corresponding to the first and second symbols. Accordingly, the linearly combined $N$-chip vector output for the first symbol, $z_{m(1)}$, and output for the second symbol $\mathbf{z}_{m(2)}$, are given by [7]

$$
\begin{aligned}
& \mathbf{z}_{m(1)}=A_{m, 1,1}^{*} \mathbf{y}_{m}^{(1)}+A_{m, 2,1}^{*} \mathbf{y}_{m}^{(2)} \\
& \mathbf{z}_{m(2)}=A_{m, 2,1}^{*} \mathbf{y}_{m}^{(1)}-A_{m, 1,1}^{*} \mathbf{y}_{m}^{(2)}
\end{aligned}
$$

where $A_{m, p, k}^{*}$ represents the complex conjugate of the channel transfer function $A_{m, p, k}=\alpha_{m, p, k} \exp \left(j \beta_{m, p, k}\right)$. Because of the harmonic and IMD terms present on each antenna path, (11) and (12) can be written as

$$
\begin{aligned}
\mathbf{z}_{m(i)} & =\sum_{p=1,2}\left\{\zeta_{m, p, 1} c_{m, p, 1}^{(i)} \mathbf{a}_{m, 1}^{+}+\sum_{\{3(m)\}} \zeta_{3(m), p, 1} c_{3(m), p, 1}^{(i)} \mathbf{a}_{3(m), 1}^{+}\right. \\
& \left.+\sum_{\{5(m)\}} \zeta_{5(m), p, 1} c_{5(m), p, 1}^{(i)} \mathbf{a}_{5(m), 1}^{+}\right\}+\mathbf{I}_{m}+\mathbf{J}_{m}+\mathbf{n}_{m}, \quad(13)
\end{aligned}
$$

where $\zeta_{m, p, 1}=\alpha_{m, p, 1}^{2}, \zeta_{I(m), p, 1}=\alpha_{m, p, 1}^{2} \cos \left(\theta_{I(m), 1}-\right.$ $\left.\theta_{m, 1}\right), \mathbf{J}_{m}$ and $\mathbf{n}_{m}$ are the output vectors due to the jamming signal and AWGN, respectively, $\mathbf{I}_{m}$ is the output vector due to the other users interference in the $m$ th branch, and is given by

$$
\begin{aligned}
& \mathbf{I}_{m}=2 \sum_{k=2}^{K} \sum_{p=1,2}\left\{\xi_{m, p, k}\left[c_{m, p, k}^{i} \mathbf{a}_{m, k}^{+}+c_{m, p, k}^{i-1} \mathbf{a}_{m, k}^{-}\right]\right. \\
& +\sum_{\{3(m)\}} \xi_{3(m), p, k}\left[c_{3(m), p, k}^{i} \mathbf{a}_{3(m), k}^{+}+c_{3(m), p, k}^{i-1} \mathbf{a}_{3(m), k}^{-}\right] \\
& \left.+\sum_{\{5(m)\}} \xi_{5(m), p, k}\left[c_{5(m), p, k}^{i} \mathbf{a}_{5(m), k}^{+}+c_{5(m), p, k}^{i-1} \mathbf{a}_{5(m), k}^{-}\right]\right\}
\end{aligned}
$$

where $\xi_{m, p, k}=\alpha_{m, p, 1} \alpha_{m, p, k} \cos \left(\theta_{m, k}+\beta_{m, p, k}-\theta_{m, 1}-\right.$ $\left.\beta_{m, k, 1}\right),{ }^{\prime} \xi_{I(m), p, k}=\alpha_{m, p, 1} \alpha_{m, p, k} \cos \left(\theta_{I(m), k}+\beta_{m, p, k}-\right.$ $\left.\theta_{m, 2}-\beta_{m, k, 1}\right)$, and $\mathbf{a}^{ \pm}$are the cross-correlation vectors after the despreader within the $i$ th symbol of the desired signal. The elements of vector $\mathbf{a}^{ \pm}$are given by

$$
\begin{aligned}
& a_{I(m), k}^{+}{ }^{(j)}=U\left(j M T_{c}-\tau_{k}\right) a_{m, 1}^{(j)} f_{I(m), k}\left(j M T_{c}-\tau_{k}\right)
\end{aligned}
$$

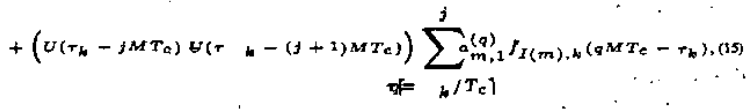

$$
\begin{aligned}
& a_{I(m), k}(j)=U\left(\tau_{k}-(j+1) M T_{c}\right) a_{m, 1}^{(j)} J_{I(m), k}\left(j M T_{c}-\tau_{k}\right) \\
& \left.\mid \tau_{k} / T_{\mathrm{c}}\right\}-1 \\
& +\left(U\left(\tau_{k}-j M T_{c}\right) \forall\left(\tau k-(j+1) M T_{c}\right)\right) \sum_{g=j}^{a} a_{m, 1}^{(q)} \tilde{f}_{I(m), k}\left(q M T_{c}-\tau_{k}\right),(10)
\end{aligned}
$$

where $U(t)$ is a unit step function. Note that

$$
\tilde{f}_{m, k}(t)=\sum_{n=0}^{N-1} a_{m, k}^{(n)} y\left(t-n M T_{c}\right)
$$

where $y(t)$ is the output of the chip matched filter. In the absence of an MMSE receiver to suppress IMD, the $N$ elements in the vector $\mathbf{Z}_{m}(i)$ are summed up, the result of which is given as soft input to the deinterleaver/Viterbi decoder [10]. Here, we propose the following MMSE receiver at the despreader output on each subcarrier to suppress the IMD and obtain an upper bound on the coded BER performance.

\section{MMSE RECEIVER}

The MMSE receiver on the $m$ th subcarrier receives the stream of $z_{m}$ vectors (we have omitted the index $i$ for notational simplicity) and chooses the tap weight vector that minimizes the conditional mean square error (MSE), conditioned on all parameters of the desired user and certain parameters of the MAI and IMD, i.e., $\left\{\mathbf{a}_{I(m), k}^{ \pm}\right\}$. The MSE is given by 


\section{Communications - $1 / / 813$}

$$
M S E=E\left\{\mathbf{w}_{m}^{\prime} \mathbf{z}_{m}-c_{m}\right\}^{2},
$$

where $\mathbf{w}_{m}$ is the $N$-element tap weight vector, $c_{m}$ is the data bit on the $m$ th subcarrier (omitting all indices in $c$ other than $m$ for notational convenience), and 'denotes transpose operation. The optimum tap weight vector $\left(\mathbf{w}_{m}\right)_{\text {opt }}$ is given by

$$
\left(\mathbf{w}_{m}\right)_{\text {opt }}=\mathbf{R}_{m}^{-1} \mathbf{g}_{m},
$$

where $\mathbf{R}_{m}=E\left\{\mathbf{z}_{m} \mathbf{z}_{m}^{\prime}\right\}$ and $\mathbf{g}_{m}=E\left\{c_{m} \mathbf{z}_{m}\right\}$. Therefore, the soft output from the $m$ th-MMSE filter is given by

$$
\tilde{c}_{m}=\left(\mathbf{w}_{m}\right)_{o p t}^{\prime} \mathbf{z}_{m},=S_{m}+I M_{m}
$$

where $S_{m}$ is desired signal component at the $m$ th MMSE filter output and $I M_{m}$ represents the undesired components due to IMD, MAI and AWGN. Note that $\left\{\tilde{c}_{m}\right\}$ output from each subcarrier MMSE filter are passed to the deinterleaver, the output of which is fed to the Viterbi decoder.

\section{A. Output SINR and Bound on Coded BER}

The desired signal component at the MMSE filter output is given by

$$
S_{m}=\left(\mathbf{w}_{m}\right)_{o p t}^{\prime} \mathbf{D}_{m}
$$

where $\mathbf{D}_{m}$, assuming rectangular chip waveform and ignoring $\mathrm{ICl}$, is given by

$$
\mathbf{D}_{m}=G_{1} c_{m} \mathbf{1}_{N}, \sum_{p=1,2} \zeta_{m, p, 1}
$$

where $\mathbf{1}_{N}$ is an $N$-dimensional vector of all ones. The undesired components at the MMSE filter output is given by

$$
I M_{m}=\left(\mathbf{w}_{m}\right)_{o p t}^{\prime}\left(\mathbf{z}_{m}-\mathbf{D}_{m}\right) \text {. }
$$

The average output signal-to-interference-plus-noise (SINR) ratio on the $m$ th subcarrier branch is then obtained as

$$
\overline{\gamma_{m}}=\frac{E\left\{\left|S_{m}\right|^{2}\right\}}{E\left\{\left|I M_{m}\right|^{2}\right\}} .
$$

From this output SINR, a bound on the coded BER performance of the system can be obtained as given in [2]. As in [2], the probability of bit error may be union bounded as

$$
P_{b} \leq\left.\frac{d T\left(D_{1}, . . D_{M}, \mu\right)}{d \mu}\right|_{\mu=1, D_{M}=1 /\left(1+\overline{\gamma_{m}}\right), m=1, \ldots, M},
$$

where $T\left(D_{1}, \ldots, D_{M}, \mu\right)$ is the transfer function of a given convolutional code. Here, we consider a rate-1/4 convolutional code with constraint length 3 whose transfer function is given by [3]

$$
\text { Z } \left.\quad{ }_{1}, D_{2}, D_{3}, D_{4}, \mu\right\}=\frac{\mu^{2} D_{1}^{4} D_{2}^{3} D_{3}^{2} D_{4}^{2}+\mu D_{1}^{2} D_{2}^{3} D_{3}^{8} D_{4}^{3}-\mu^{2} D_{1}^{2} D_{2}^{4} D_{3}^{4} D_{4}^{4}}{1-2 \mu D_{2} D_{3} D_{4}-\mu^{2} D_{1}^{2}+\mu^{2} D_{2}^{2} D_{3}^{2} D_{4}^{2}}
$$

Substituting (26) into (25) results in the upper bound on the probability of bit error. For a rate $=1 / 2$ convolutional code with constraint length 3 , the transfer function is give by [3]

$$
T\left(D_{1} H Q\right)-\frac{\mu D_{1}^{2} D_{2}^{3}+D_{1}^{4} D_{2}^{2} \mu^{2}-D_{1}^{2} D_{2}^{4} \mu^{2}}{1-2 D_{E} \mu{ }_{1}^{2} \mu^{2}+D_{2}^{2} \mu^{2}}
$$

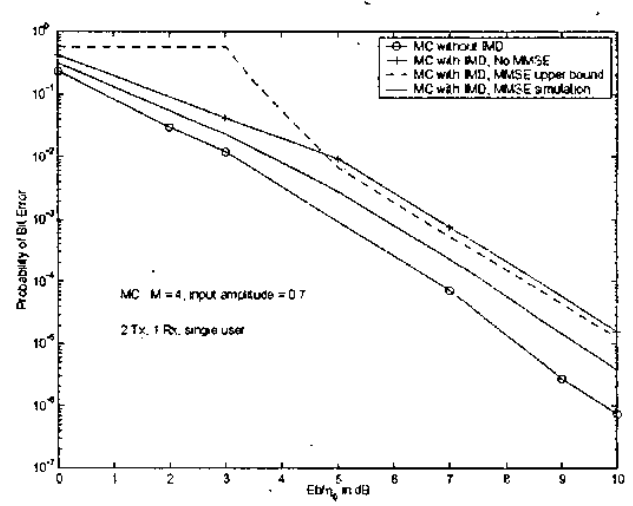

Fig. 3. Probability of bit error in the presence of IMD without and with MMSE receiver. $P=2, M=4, K=1$.

\section{PERFORMANCE RESULTS}

In this section, we present the numerical results of the coded bit error rate (BER) performance of the system in the presence of IMD with and without the MMSE receiver. Fig. 3 shows the coded BER as a function of $E_{b} / \eta_{0}$ for the single user case $(K=1$ ), for number of transmit antennas $P=2$, number of subcarriers on each transmit antenna $M=4$, and rate $-1 / 4$ convolutional code of constraint length 3 . The input amplitude to the PA is taken to be 0.7 per subcarrier. Fig. 3 shows the performance without IMD (which gives the best possible performance) as well as with IMD (because of which performance degrades). In the presence of IMD, the performance without and with MMSE receiver are plotted. We show both the upper bound on the coded BER obtained using (25) as well as the coded BER obtained from bit error simulations: As expected, in the presence of IMD with no MMSE, the performance degrades compared to that without IMD. It is seen that when the MMSE filter is used, the performance improves significantly because of the ability of the MMSE filter to suppress the IMD components. The bound is found to be reasonably close to the simulation results for large SNRs and loose at low SNRs, which is expected from the union bounding.

In Figs. 4 to 6, we compare the performance of two systems, namely, a ( $P=2, M=4)$ system with with rate-1/4 coding (i.e., $2 \mathrm{Tx}$ antennas and 4 subcarriers on each Tx antenna), and a $(P \notin, M=2)$ system with rate-1/2 coding (i.e., 4 Tx antennas, 2 subcarriers on each Tx antenna), in the presence of IMD without and with the MMSE receiver. Note that the diversity order (8th-order diversity) and the system bandwidth $(W)$ in both these systems are kept the same. A comparison of the performance of these two systems, without coding and no IMD suppressing MMSE receiver, was presented in [10], where it was shown that a) in the absence of IMD and jamming, both $(P=2, M=4)$ and $(P=4 . M=.2)$ systems performed identical (because of same diversity order), b) in the presence of jamming alone and no IMD, the ( $P=2, M=4$ ) system performed significantly better than the $(P=4, M=2)$ system (because more the number of subcarriers better is the performance under jamming conditions), and c) in the presence of both IMD and jamming; the ( $P=2, M=4$ ) system loses its performance due to severe IMD effects. Our intent here is to suppress the IMD effects us- 


\section{TENCON $2003 / 814$}

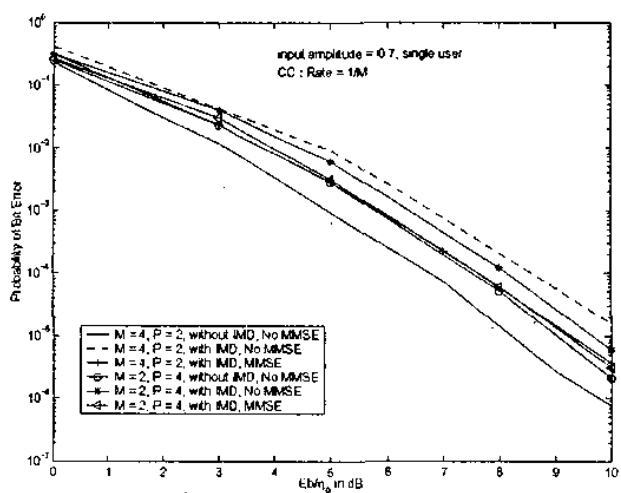

Fig. 4. Probability of bit error in the presence of IMD without and with MMSE receiver for $a)(P=2, M=4)$ system and $b)(P=4, M=2)$ system. $K=1$

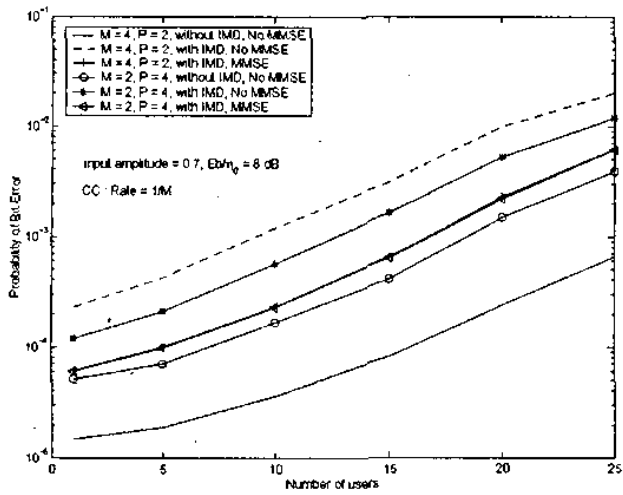

Fig. 5. Probability of bit error $v s$ number of users in the presence of IMD without and with MMSE receiver for $a)(P=2, M=4)$ system and $b)$ $\left(r-4, M_{-}<\right.$) system. $\boldsymbol{c}_{b} / y_{0}-\mathrm{adB}$.

ing the MMSE receiver and reduce the loss due to IMD, while retaining better antijamming performance. Our results in Figs. 4 to 6 illustrate that this intent has been achieved effectively.

Fig. 4 shows the single user performance as a function of $E_{b} / \eta_{0}$ in the presence of IMD, without and with MMSE receiver. The performance without IMD and no MMSE is also plotted for comparison. The difference between the performance of the $(P=2, M=4)$ and the $(P=4, M=2)$ systems, for the case without IMD and no MMSE, is due to the coding gain difference between rate- $1 / 4$ and rate- $1 / 2$ coding schemes used, with ( $P=2, M=4$ ) scheme performing better due to rate-1/4 code used). However, in the presence of IMD, it is observed that without MMSE, the $(P=2, M=4)$ system performs worse than the $(P=4, M=2)$ system, because of a greater loss due to IMD effects in the $(P=2, M=$ 4) system. However, when the proposed MMSE receiver is used in the presence of IMD, the $(P=2, M=4)$ system performs as good as the ( $P=4, M=2$ ) system, essentially suppressing IMD and compensating for the loss due to IMD effects. A similar performance gain due to IMD suppression by the MMSE receiver is also observed in the presence of multiple users (MAI) as illustrated in Fig. 5. More importantly, while the ( $P=2, M=4)$ system with MMSE behaves as good as the ( $P=4 \cdot M=2$ ) system, its antijamming performance is much superior compared to that of the $(P=4, M=2)$ system, which is illustrated in Fig. 6 .

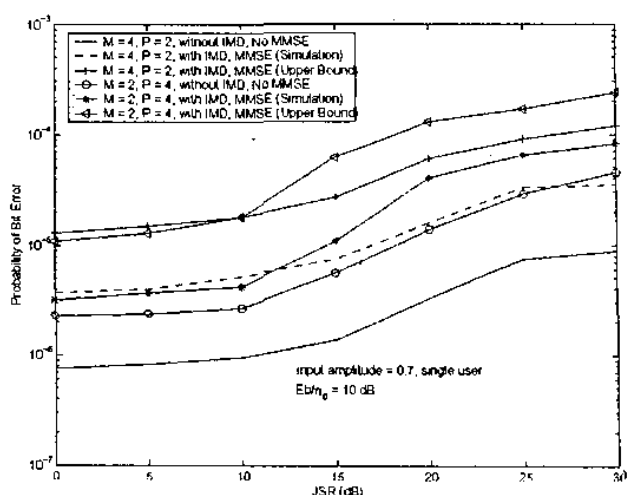

Fig. 6. Probability of bit error $v s$ JSR in the presence of IMD without and with MMSE receiver for $a)(P=2, M=4)$ system and $b)(P=4, M=2)$ system. $E_{b} / \eta_{0}=10 \mathrm{~dB}$.

Fig. 6 shows the coded BER performance as function of jamming signal-to-signal ratio (JSR). The jamming signal bandwidth is taken to be $W, 4 \mathrm{~Hz}$ which is centered around $3 W$. It is observed that in the presence as well as in the absence of IMD, the ( $P=2, M=4)$ system offers superior performance compared to ( $P=4, M=2$ ) system, particularly at high JSR conditions. Thus, the proposed MMSE scheme effectively suppresses IMD and reduces the performance loss due to IMD, while retaining better performance under jamming conditions.

\section{CONCLUSIONS}

We presented a design of a multicarrier MMSE transceiver to suppress intermodulation distortion introduced by the nonlinear power amplifier in a DS-CDMA system using multiple transmit antennas. The results demonstrate that the proposed coded MC MMSE system can offer better performance than does the system without MMSE in the presence of IMD MAI and jamming, and also achieve coding gain, without requiring knowledge of other users' spreading codes, timing or phase.

\section{REFERENCES}

[1] S. Kondo and L B. Milstein, "Performance of multicarrier DS-CDMA systems," IEEE Trans, Commun.. pp. 238-246, February 1996.

[2] D. N. Rowitch and L. B. Milstein, "Convolutionally coded multicarrier DS CDMA systems in a multipath fading channel - Part I: Performance analysis," IEEE Trans. Commun. vol. 47, pp. 1570-1582, October 1999.

[3] W. Xu and L. B. Milstein, "On the use of interference suppression to reduce intermodulation distortion in multicarrier CDMA systems," IEEE Trans. Commun., vol. 49 , no. 1, pp. 130-141, January 2001

[4] K. R. Shankar kumar and A. Chockalingam, "Performance of multicarrier DS-SS with imperfect carrier phase on multipath Rayleigh fading channels," Proc. IEEE VTC'02 (Fall), Vancouver, September 2002.

[5] J. C. Fuenzalida, O. Shimbo, and W. L. Cook, "Time-domain analysis of intermodulation effects caused by nonlinear amplifi ers," COMSAT Tech. Rev. vol. 2, no. 1, pp. 89-143, Spring 1973.

[6] V. Tarokh, N. Seshadri, and A. R. Calderbank, "Space-time codes for high data rate wireless communications: Performance criterion and code construction," IEEE Trans. Inform. Theory, pp. 744-765, March 1998.

[7] S. Alamouti, "A simple transmit diversity technique for wireless communications," IEEE Jl. Sel. Areas Commun. pp. 1451+1458, Oct. 1998.

[8] V. Tarokh, H. Jafarkhani, and A. R. Caldertank, "Space-time block codes from orthogonal designs," IEEE Trans. Inform. Theony, vol. 45, no. 5 , pp. 1456-1467, July 1999.

[9] Q. H. Spencer and A. L. Swindlehurst, "On the performance of multicarrier CDMA using multiple transmitters," Proc. IEEE ICA4SP'01, pp. 2397-2400, May 2001.

[10] K. R. Shankar kumar and A. Chockalingam, "Effect of amplifi er nonlinearity and jamming in multicarrier DS-SS systems with multiple transmit antennas," Proc. National Conf. on Commun.. pp. 85-89, IIT, Chennai, January 2003. 